ISSN: $1130-3743$

\title{
LA FUNCIÓN DE LA UNIVERSIDAD. DE LA ESTRATEGIA POLÍTICA A LA POLÍTICA ESTRATÉGICA
}

\author{
The function of the university. From policy strategy to stra- \\ tegic policy
}

\author{
José Manuel TOURIÑÁN LÓPEZ, Miguel Ángel SANTOS REGO, Antonio RODRÍGUEZ \\ MARTÍNEZ y María del Mar LORENZO MOLEDO \\ Universidad de Santiago de Compostela. Facultad de Ciencias de la Educación. Departamento \\ de Teoria e Historia de la Educación. Campus Sur s/n., 15706 Santiago de Compostela
}

BIBLID [(1130-3743) 10, 1998, 13-35]

\section{RESUMEN}

En este artículo examinamos la compleja relación entre política y universidad, dos conceptos con significado propio y que sólo en parte se fusionan respecto de la función de la universidad, que es el objeto principal de este estudio.

Una vez delimitado el sentido de la contribución abordamos el marco de la coordinación competencial en nuestro país, junto con las directrices de la educación superior en el marco europeo y el énfasis actual en la búsqueda de la calidad universitaria como vía de desarrollo estratégico. Es justamente la cultura de la evaluación en la educación superior la que favorece un renovado análisis de la calidad en el contexto de una universidad de masas.

Acorde con el notable reto derivado del Tratado de Maastricht, nos ocupamos del desarrollo de sistemas universitarios regionales y las políticas estratégicas asociadas a la definición de perfiles en las universidades. Finalmente, referimos algunos elementos importantes para una articulación de funciones que la universidad tendrá que plantearse en el nuevo siglo.

\section{SUMMARY}

The main objective of this article is to examine the complex relationship between policies and the university, two concepts, each with their own meanings that fuse only in part, with respect to the function of the universities themselves. 
Once the sense of this article has been established, we will analyse the framework given to the co-ordination of what should be done, why and by whom at the different Spanish universities. We will also analyse the guidelines for higher education within the European framework and the current emphasis given to the search for quality in universities, as a means to achieving a strategic development. It is precisely the "culture" of evaluation in higher education that helps towards a renewed analysis of this quality within the context of mass universities.

In correlation with the important challenge derived from the Maastricht Treaty, we will deal with the development of regional university systems, as well as the strategic policies related to the definition of university profiles. Finally, we make reference to some important elements for the articulation of functions which universities will have to face in the next millennium.

\section{INTRODUCCIÓN}

Dialogar sobre política y universidad implica la necesaria clarificación de cuestiones importantes acerca de la vida pública y de la democracia participativa, difícilmente agotables en este artículo que pretende acercarnos determinadas ideas y pautas estratégicas de análisis en las que podamos reconocer algunos hechos y retos que van asomando por las puertas de la institución en la relación política y universidad ante la cuestión de la función de la universidad.

La universidad como problema y también como solución es simplemente una referencia conceptual y filosófica, con toda su carga simbólica y su expresividad cultural a lo largo de las épocas. No en vano se trata de una institución comprensible según modelos que hunden sus raíces en determinadas visiones del mundo y del hombre (Matteucci, 1991; Sánchez-Salorio, 1994), felizmente tensionada en su historia por la febril apuesta entre paradigmas, con ensamblajes político-administrativos en absoluto ajenos a las respectivas tradiciones y "ethos" culturales, pero casi nunca insensible a los cambios sociales y económicos de las centurias pasadas y de esta que ya va feneciendo.

La universidad es parte sustantiva, pero sólo parte al fin, de la sociedad. La mutua imbricación es desigual y aún asimétrica en no pocos de sus contornos. Es indudable que la política de gobierno general influye mucho más sobre la universidad que lo que es el caso en sentido inverso (Barber, 1998), pero también es cierto que la universidad es uno de los escenarios donde con más fuerza se proyectan recurrentes inquietudes y contradicciones sociales o, diciéndolo de otra forma, se representa con toda suerte de matices la gama de anhelos que circundan un diagnóstico crítico de la comunidad en su conjunto. Tal vez encuentre aquí acomodo la persistente noción de que la educación universitaria contribuye, en cierto modo, a elevar la "sabiduría" política y moral (ver O'Brien, 1998).

Puesto que, en apreciable medida, reflejamos el tipo de sociedad en la que estamos instalados, es obvio colegir que nuestra institución participa de aquellas virtudes, pero también de los vicios que, al menos sobre el papel, expresan tópicamente un estado de cosas en el ambiente. En tal sentido, es singularmente curioso reparar en la presencia de un sistemático correlato, dentro del sistema 
social imperante en lo que llamamos Occidente, entre formas organizativo-administrativas en las instancias de gobierno político general y representación de poder en el organigrama universitario. El proceso que rige la toma de decisiones pone de manifiesto tan ostensible parangón. Justamente el conocimiento de las diferencias por países y sistemas universitarios es el que nos permite comprender las mayores o menores cotas de funcionalidad y de responsabilidad en la decisión.

En la sociedad actual nadie pone en duda el valor de la inversión en capital humano, el valor económico de la educación, la importancia de la subsidiariedad, la necesidad de autonomía en la universidad, el rendimiento efectivo de la institución universitaria y la responsabilidad social compartida acerca de la universidad (Touriñán, 1993a).

La universidad se predica como un recurso tecnológico de primer orden y su desarrollo es un instrumento estratégico de innovación. De ahí que, en el marco de este trabajo, el estudio, la docencia, la investigación, la profesionalización, la cultura y la conciencia social crítica -que son funciones inherentes al concepto de universidad- tengan que ser integradas o reinstaladas dentro del marco de la relación entre política y universidad.

El indicador básico de ese nexo es el modo en que se resuelve en cada caso concreto el juego de la relación entre autonomía universitaria y coordinación; porque la universidad tiende a sus fines $y$, habida cuenta de las competencias que correspondan a las administraciones, así como del valor estratégico de la universidad, la prestación de un servicio social, que es público, puede convertirse en el objetivo de la pública intromisión en el derecho de la Academia a disponer y generar, rindiendo cuentas, un espacio para la docencia y la investigación de calidad.

En el fondo, la crisis permanente de la universidad, tiene siempre que pasar por la lúcida barrera conceptual orteguiana; la crisis no es sinónimo de decadencia, ni de simple necesidad de corrección de abusos. La crisis en la universidad es sinónimo de generación de nuevos usos porque, necesariamente, para cumplir su misión tiene que estar a la altura de los tiempos en sus cambiantes circunstancias. Éste es el reto de la universidad, aunque sólo sea para no dejarse arrebatar lo que debe seguir siendo un irrenunciable puesto de mando en la dinámica de innovación y de conocimiento en esta sociedad de la información, ya digitalizada, con nuevos marcos y relaciones estratégicas en el mundo del trabajo, pero sobre todo asistiendo a una imparable revisión de valores en una marcha cultural, tecnológicamente instigada, que reclama nuestro protagonismo; con apoyo social, pero también con nuevas ideas, despues de Maastricht (Touriñán, 1996a; Huntington, 1997; Quintanilla, 1998).

\section{POLÍTICA Y UNIVERSIDAD: EL MARCO DE LA COORDINACIÓN COMPETENCIAL}

En nuestro país se diferencia, administrativamente hablando, educación superior y educación universitaria. Sobre esa base, es legítimo decir que, en líneas 
JOSÉ M. TOURIÑÁN, MIGUEL Á. SANTOS, ANTONIO RODRÍGUEZ Y M. a DEL MAR LORENZO LA FUNCIÓN DE LA UNIVERSIDAD. DE LA ESTRATEGIA POLÍTICA A LA POLÍTICA...

generales, la circunstancia más peculiar del marco legal vigente es que la Ley de Reforma Universitaria (LRU) se aprobó pensando en una realidad universitaria (la específica del año 1983) y que esa realidad legislada se modificó posteriormente con el proceso de transferencia de competencias a las Comunidades. Es decir, que la legislación actual permite elaborar la política universitaria desde tres instancias: el Estado, las Comunidades Autónomas (que tienen transferidas las competencias en materia de educación superior y universidades) y las universidades, como se desprende de los artículos 2 (derecho a la autonomía de las nacionalidades y regiones) y el 27.10 (reconocimiento de la autonomía universitaria) de la Constitución Española y del artículo 3.2 de la LRU, que establece las competencias propias de la autonomía universitaria.

Así pues, la existencia de estos tres niveles distintos éstá diseñada teóricamente para respetar la autonomía universitaria, entendida ésta como capacidad de autonormación, y postula, como sabemos, una exigencia de coordinación en dos niveles: el Consejo de Universidades, a nivel nacional, y el Consejo Universitario de cada Autonomía con competencias en materia de universidades. Cabe afirmar, sintéticamente, que el Sistema Universitario, desde la perspectiva de la política universitaria, está diseñado para tratar de conjugar el respeto a la autonomía de cada universidad y la voluntad de ejercer las competencias propias de las Administraciones (Touriñán, 1993).

Por otra parte, creemos que esta situación general del marco legal está especialmente afectada por el proceso de transferencias hasta tal extremo de que, suponiendo la LRU un marco legal anterior a las transferencias, se hacen patentes las dificultades para coordinar el sistema universitario una vez acabado el proceso de transferencia de las universidades. Conviene, pues, introducir racionalidad en los procesos de creación y desarrollo de un Sistema Universitario, desde unas competencias "compartidas" (término más adecuado que el eufemismo de "plenas"), hoy por hoy atadas por un marco legal manifiestamente mejorable, pensando en los conceptos básicos de racionalidad, calidad y competitividad o eficacia, que son los que marcan en este fin de siglo las mejores expectativas de un proyecto universitario. Es claro que, como consecuencia de los procesos de transferencia de competencias, el sistema de enseñanza superior en España se ve por:

- La disminución y remodelación cuantitativa y cualitativa de las funciones del Ministerio de Educación.

- La posible descoordinación entre las distintas Comunidades con competencias transferidas e incremento de la dificultad de realizar una planificación nacional de la enseñanza superior.

- La posibilidad de ir aumentando poco a poco la diferenciación entre universidades en las distintas Comunidades Autónomas.

Naturalmente, la descoordinación entre Comunidades puede ser el fruto temprano del ejercicio de la autonomía en materia de educación. Pero esa actuación está llevando a duplicidades en la necesidad de recursos y a una falta de especialización, con el peligro de que el proceso de desarrollo de cada Comunidad Autónoma llegue a convertirse en un proceso mimético, sin nítida visión de futu- 
ro, y con un alto grado de vulnerabilidad ante las universidades de otros países, por no tener una conciencia de identidad particular de la enseñanza superior en cada Comunidad. Esta dificultad para llevar a cabo una planificación de la enseñanza superior en España podría provocar, si no se establecen mecanismos de control y coordinación adecuados, un desajuste entre la oferta global y la demanda que va a afectar a la política general de movilidad estudiantil.

Las realidades internas y externas de hoy están presionando para modificar la universidad y deshacer sus deficiencias. Con el objeto de hacer frente a las mismas se están activando a nivel nacional diversos mecanismos que se dirigen a modificar la institución, siendo posible referir cuatro líneas de acción al respecto:

1) La reorganización general de la enseñanza mediante la reforma de los planes de estudios.

2) La evaluación de la calidad de las universidades y del rendimiento del profesorado.

3) Los nuevos mecanismos de financiación.

Tales acontecimientos y observaciones son manifestaciones diversas de una idea genérica de crisis de gestión en la universidad (Touriñán, 1997) que se enfrenta ahora de manera muy particular, no a la corrección de abusos, sino a la creación de nuevos usos en un ámbito de responsabilidad compartida, que además es un ámbito de exigencias diferenciadas.

\section{LAS DIRECTRICES DE LA EDUCACIÓN SUPERIOR EN EL MARCO EUROPEO}

Recordemos de entrada que el Tratado de la Unión Europea (TUE) supuso la segunda gran revisión a la que se vió sometida la Comunidad Europea. La primera, desde su construcción, fue el Acta Única Europea (AUE) de 1986, que consagró la llamada "Europa de los mercaderes" al abrir la puerta de los doce países socios en ese momento al mercado único.

Con esta segunda gran revisión, por medio del TUE, pueden destacarse cinco presupuestos de interés para el contexto educativo (Touriñán, 1997b):

a) Reafirmación de la voluntad de una política común más allá de lo económico. No en vano el Tratado de Maastricht suprime de la CE el calificativo de "económica", permanente desde los acuerdos de 1957.

b) Promoción de la educación de calidad a través de la cooperación entre los Estados miembros. Cierto que la mención a la educación es corta pero lo importante es que aparece por vez primera un capítulo educativo en texto comunitario de tan alto significado.

c) Soberanía de los Estados y Subsidiariedad. Relevancia de la coordinación y política de progreso social bajo el principio de asociación cooperativa de los estados miembros, los interlocutores sociales y los ciudadanos, cuya clave debe ser el concepto activo y positivo de la subsidiariedad (ver Santos Rego, 1997). Es decir, la UE emprenderá una acción si los objetivos no pueden ser alcanzados suficientemente por (o dentro de) los propios Estados miembros. 
d) El procedimiento de la cooperación se aplicará, gracias al TUE, tanto a las disposiciones del fondo social y regional como a la formación profesional y a la cooperación para el desarrollo, a la política social, al medio ambiente y al mercado de trabajo. Desde la perspectiva de la educación, el procedimiento abre un lugar específico para el consenso, que estará en las bases de las estrategias de persuasión, cooperación y conciliación entre los Estados miembros.

e) El TUE no es un documento definitivo en su contenido. De hecho, ya se ha producido su revisión en 1996, una vez producida la incorporación efectiva de tres nuevos socios: Austria, Finlandia y Suecia.

Es el título VIII de la tercera parte del TUE, dedicado a las políticas sociales, el que establece en su capítulo tres las directrices comunes a la educación, la formación profesional y la juventud. El título XV de esta tercera parte se dedica a investigación y desarrollo tecnológico.

Así pues, educación e investigación constituyen los dos ejes desde los que se construyen en la UE las directrices para la enseñanza superior. Los contenidos conceptuales básicos que aparecen en el texto articulado abarcan temas como la educación de calidad, el fomento de la cooperación entre Estados, el respeto a los sistemas educativos de los Estados y la diversidad cultural y lingüística, el fomento de la movilidad y el intercambio, el desarrollo de la dimensión europea de la educación, el fomento y desarrollo de la educación a distancia, el desarrollo de la competitividad, la estimulación de la IDT de alta calidad, así como la difusión y explotación de resultados de investigación y transferencia de tecnología (Comisión de las Comunidades Europeas, 1993, 1994)

Estos conceptos, que forman parte del contenido de los artículos 118, 126, 127 y $130 \mathrm{~F}$ conforman el sistema conceptual presente en los "libros blancos" que la UE ha impulsado en el contexto de la educación y la investigación. Nos referimos ahora al libro blanco de "crecimiento, competitividad y empleo" y al de "política social europea", destacando los siguientes principios generales:

- El empleo es la clave de la integración social y económica (Política Social).

- La competitividad y progreso social son dos caras de la misma moneda (Política Social).

- La convergencia de objetivos respeta la diversidad (Política Social).

- La armonización total de políticas sociales no es un objetivo de la Unión (Política Social).

- La creación de servicios básicos transeuropeos y el desarrollo y formación en nuevas tecnologías es fuente de competitividad (Crecimiento).

- La libre circulación de trabajadores obliga a plantear de manera prioritaria la correspondencia de cualificaciones y el reconocimiento mutuo de títulos (Política Social).

- El fomento de la cooperación entre las políticas de investigación de los países miembros y las empresas es la garantía para tener en cuenta las potencialidades del mercado (Crecimiento).

Coincidiendo el grupo con una perspectiva ya expresada (Touriñán, 1997b; Santos Rego, 1997a), afirmamos que el TUE sienta las bases para la construcción 
de un proyecto educativo común, aceptando y asumiendo la pluralidad nacional existente a través de la coordinación de tareas en la construcción de la Unión. En otras palabras, un proyecto de integración no excluyente de la diversidad y respetuoso con la soberanía de cada Estado.

Con el TUE, los millones de estudiantes de los 15 Estados de la Unión afrontan una situación general de los sistemas de enseñanza superior europea caracterizada en común por la diversidad lingüística, la diversidad administrativa y estructural de sistemas, la diversidad de situaciones y niveles de desarrollo socio-económico y la diversidad de lugares y exigencias (Malosse, 1994; Touriñán, 1997c; Santos Rego, 1997). En tal situación, la tendencia sigue apuntando a la expansión cuantitativa, que se ve acompañada por restricciones financieras que obligarán a las partes a plantearse, en palabras de la Unesco, un pacto académico en el que las universidades logren un desarrollo humano sostenible a través de la educación.

Puede decirse que el marco educativo de la UE se corresponde con un ente descentralizado que postula la autonomía escolar, la libertad académica y la identidad europea definida dentro de sistemas educativos estatales, regionales y locales diversos.

Junto con la profesionalización, la descentralización educativa (que se traduce en fórmulas organizativas que postulan la convergencia desde la diversidad) aparece como el acontecimiento estructural más relevante en el análisis socio-político de los sistemas educativos. La descentralización reclama la existencia de órganos de gobierno con competencias en cada territorio, pues una organización descentralizada no es simplemente lo que acerca el poder a cada demarcación de influencia, sino la que desarrolla una organización horizontal del poder con lazos de interdependencia coordinados bajo el principio de atribución territorial de autonomía de decisión.

Del mismo modo, no sería muy realista trasvasar las experiencias concretas de descentralización de un país a otro. Cada alternativa de descentralización nace en una circunstancia socio-histórica desde la que se debe construir la posición descentralizada de equilibrio siempre entre elementos antinómicos que, por tener ese carácter, son imprescindibles. Touriñán (1995) ha identificado los siguientes elementos antinómicos de los sistemas educativos descentralizados:

- La alternativa regionalización-internacionalización.

- La alternativa heterogeneidad-homogeneidad.

- La alternativa diversificación cultural-equivalencia internacional de sistemas educativos.

- La alternativa autoidentificación del sistema en la Comunidad Regional-uniformidad inter e intra Comunidad.

- La alternativa determinación aislada del sistema en la Comunidad Regionaldesarrollo educativo solidario entre Comunidades.

Este equilibrio de opciones antinómicas forma parte sustantiva de la alternativa de descentralización, que se ve delimitada por dos extremos: la uniformidad total de un centralismo utópico y el aislamiento o cierre sobre sí mismo de la descentralización radicalizada. La solución ha de encontrarse en las sinergias y líneas 
JOSÉ M. TOURIÑÁN, MIGUEL Á. SANTOS, ANTONIO RODRÍGUEZ Y M.․ DEL MAR LORENZO

LA FUNCIÓN DE LA UNIVERSIDAD. DE LA ESTRATEGIA POLÍTICA A LA POLÍTICA...

de convergencia que reconozcan el desarrollo de los sistemas universitarios y de las comunidades científicas como instrumentos de innovación y desarrollo.

Hace ya seis años que se marcaron, con motivo de un coloquio sobre la enseñanza superior pensando en el 2000 (EOLAS, 1992) unas áreas decisivas para la educación universitaria europea. Dichas áreas abarcan desde la participación y acceso a la enseñanza superior hasta la dimensión europea de la misma, pasando por la colaboración con el mundo empresarial, la formación continua, y la educación a distancia. Especialmente interesante aparecía la dimensión europea de la enseñanza superior, incluyéndose ahí, entre otras, la movilidad de los estudiantes, la cooperación entre instituciones, la idea de Europa en el programa de estudios, la importancia capital de los idiomas, la formación del profesorado, el reconocimiento de cualificaciones y períodos de estudios, el análisis de la información y de las políticas, o la convergencia de programas.

\section{Política de CALIDAD Y UNIVERSIDAD DE MASAS}

En los últimos tres o cuatro años han proliferado no sólo publicaciones, sino eventos académicos y foros de distinta índole articulados en su propósito de convocatoria alrededor de las preguntas, y no siempre coincidentes respuestas, acerca de cómo plantear y conseguir más calidad en la universidad.

En nuestra opinión, estamos ante una corriente en absoluto ajena al final de un modelo y la irrupción de otro más identificado con lo que se ha convenido en llamar "la gestión estratégica y de calidad", donde asoman, naturalmente, valores, objetivos y procedimientos distantes y distintos de los convencionalmente establecidos en los pabellones de gobierno universitarios. A esto se han referido la veintena de ponencias que han circunvalado el programa del muy reciente Seminario Internacional sobre "dirección estratégica y calidad de las universidades" (Universidad Politécnica de Cataluña, enero de 1998).

Recogiendo el propio marchamo del evento, las estrategias de avance han de pasar por dibujar y lograr un marco de realidad (virtual y no virtual) propicio al diseño instrumental y evaluación de la calidad en aquello que hacemos en nuestro trabajo. Calidad al servicio de la sociedad, reza el título del documento que sobre el particular acaba de hacer público la antes citada Universidad Politécnica de Cataluña. Planteada como "misión", concita una voluntad de solvencia y excelencia cuyos objetivos no pueden ser supeditados sin más, cual letanía paralizadora, a las tasas de crecimiento en estudiantes, personal docente, no docente, etc.

Es preciso liderar el reto y solución de problemas dentro de la institución, pero también es preciso convertir a la universidad en punto de inevitable referencia dentro de la comunidad social. Esa capacidad de liderazgo está presente hoy, y lo estará mucho más, en el sentido de la calidad que, nos guste o no, se vislumbra parcialmente en torno a nuestros activos culturales y científicos de competitividad estructural, departamental y personal.

Estas propuestas, como ha apuntado el profesor Touriñán (1997a) nacen del reconocimiento de la universidad como servicio social público, que tiene como cliente al estudiante y, al mismo tiempo, a la sociedad concienciada de la impor- 


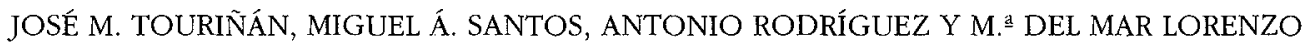
LA FUNCIÓN DE LA UNIVERSIDAD. DE LA ESTRATEGIA POLÍTICA A LA POLÍTICA...

tancia de rendir cuentas en la cultura de la calidad total en el sistema universidadsociedad-empresa y en el sistema ciencia-tecnología-sociedad.

Así pues, la exigencia de calidad parece convertirse en estandarte emblemático, referente de una nueva cultura y política universitaria de los países desarrollados. En cada lugar y contexto, según su historia, condiciones y circunstancias, este y no otro es el objetivo a definir y perseguir en el sistema, entre otras razones, porque sin mejorar la calidad no podremos disponer de elementos de juicio en la adopción política -y no simplemente tecnocrática- de las decisiones que convengan, para que la sociedad sepa del rendimiento académico y científico de los recursos que nos proporciona.

Es edificante, por otra parte, que entre los resultados del grupo de trabajo sobre "Universidades y Poderes Públicos", establecido a raíz de la reciente crisis doméstica Ministerio de Educación y Cultura-Rectores, se afirme con rotundidad (punto 5) "la extensión del principio de calidad, como exigencia básica para la evaluación docente e investigadora de las universidades, al nivel de aptitud y actitudes por parte de los estudiantes que se incorporan al sistema, lo que con toda certeza representará un reexamen en profundidad de los criterios de acceso a la universidad y de permanencia en ella, así como de las ofertas correspondientes a otras modalidades de enseñanza profesional superior no universitaria que se puedan presentar a la ciudadanía. Valga la cita del importante documento consensuado para poner de relieve el sentido extensivo que se está imponiendo al hablar de calidad en el territorio español, con una trascendencia y alcance que va más allá de las aulas.

Idéntico énfasis fue el transmitido, a nivel supranacional, por el interesante documento de política para el cambio y el desarrollo en la educación superior (Unesco, 1995). Proclamando una "universidad pro-activa" firmemente anclada en las circunstancias locales, pero plenamente comprometida en la búsqueda universal de la verdad y el progreso del conocimiento, la calidad ocupa sitial de honor entre los desafíos planteados dentro del proceso de cambio, junto a la pertinencia (papel y lugar de la educación superior en la sociedad, abarcando programas, contenidos, equidad, responsabilidad, libertad académica, autonomía institucional, etc.) y a la internacionalización (aumento de la movilidad docente y discente, comprensible a la luz de la importancia estratégica de la comunicación intercultural en una sociedad dominada por las tecnologías de la información).

Las facetas de la calidad universitaria difícilmente pueden abarcar otras que no estén subsumidas en el buen hacer de la enseñanza (calidad del personal docente y de los procesos de selección, de los programas y, como corolario, del aprendizaje), en la investigación (calidad de los proyectos, de los programas de tercer ciclo...) en la gestión y administración de los recursos, en las infraestructuras, etc., (para un análisis de este punto en perspectiva europea, ver Vorbeck, 1998). Pero veamos, antes de adentrarnos en aspectos más concretos sobre el tema de la calidad, algunas directrices surgidas en el entorno comunitario.

Es a partir de finales de los sesenta y comienzos de los setenta cuando la transformación socio-económica de España y una "nueva" política escolar (Ley General de Educación) conducen progresivamente a una sustitución de la uni- 
versidad tradicional por la universidad dual que hoy tenemos: una universidad minoritaria, con cierto influjo en determinadas élites, y una universidad pública y más masificada que de masas (Jerez Mir, 1997) para un tercio de la cohorte juvenil entre 18 y 24 años de edad. Una consecuencia de este dualismo universitario puede ser la posibilidad de que se produzca un desajuste en las oportunidades formativas y laborales de estudiantes y graduados en el sistema educativo del país, desde la perspectiva de la calidad de la educación y la inserción profesional-laboral.

Permítasenos indicar algunos datos. Terminando la década de los ochenta, el número de entradas anuales en la educación terciaria dentro de la Unión Europea se estimaba en 1,7 millones de personas, de las que 1,1 millones (aproximadamente, el 65\%) optaban por matricularse en la universidad. Actualmente, la cantidad de estudiantes matriculados en la UE en "tertiary education" supera los ocho millones. Mientras Francia y Alemania han sobrepasado los 1,5 millones, España se encuentra en ese umbral, y países como Inglaterra e Italia tienen bastante más del millón (De Woot, 1998; Consejo de Universidades, 1995; Tiana Ferrer, 1998; Uriel et al., 1997).

Hoy por hoy, a pesar del descenso demográfico, es firme la convicción acerca de la permanencia en niveles muy altos de presión por acceder a la enseñanza universitaria. Las proyecciones de la Unesco mencionan la magnitud de ciento veinte millones de jóvenes buscando educación superior hacia el año dos mil cuarenta, una gran parte de ellos ubicados en países en vías de desarrollo.

Puede hipotetizarse que, hasta cierto punto, la educación superior continuará destacándose como factor de movilidad social ascendente $\mathrm{y} / \mathrm{o}$ como elemento de movilidad profesional horizontal. Como dice el informe de la Unesco del año 95 son muchos los motivos para considerar que los estudiantes son muy beneficiosos en una sociedad, pero es igualmente oportuno entablar un debate sobre el acceso en esta era de educación superior masiva. Confesamos nuestra inquietud al respecto pensando en el tema de la equidad y las posibilidades de participación en programas de calidad por parte de grupos en situación de inferioridad social.

Conviene, por lo tanto, reparar en la necesidad de plantear los verdaderos retos de una universidad de masas, teniendo en cuenta además la reorientación tecnológica creciente en los campus, que rompe la tradición clásica del proceso de aprendizaje en los mismos (Neave, 1995). Entonces, de lo que se trata es de afrontar el problema de una educación de masas en la universidad, cuya tesis de fondo es, sencillamente, la cultura de masas y la sociedad red en un contexto económico cada vez más interconectado (Santos Rego, et al., 1996; Castells, 1997).

La aparente contradicción entre la necesidad de una educación superior masiva (en términos biográficos, ir a la universidad casi es ya una especie de urito de pasaje" como otros en la vida) y la exigencia de calidad, que algunos categorizan como "excelencia", es objeto de enfoques que emergen desde posiciones interpretativas no necesariamente contrapuestas pero sí desiguales.

En nuestra opinión, es imprescindible un compromiso político-social ante el tema, junto con mejores acentos y mayor receptividad a la investigación educativa que haya dicho o tenga aún algo importante que añadir al respecto. Defendemos 
una perpectiva desarrollada por Touriñán (1997) distinguiendo el problema de la educación de masas y el problema de la masificación en la universidad; y estableciendo como tesis de partida la ruptura de equivalencia entre educación de masas y educación de baja calidad. La educación de masas, bien entendida, cumple las exigencias del derecho a la educación, más allá del mito del igualitarismo (Touriñán, 1995) que no consiste en favorecer sólo a una minoría intelectualmente selecta, sino en ayudar a una población estudiantil numerosa que está expuesta a la masificación y, a pesar de ello, tiene que prepararse eficazmente para competir en el mundo y elegir su proyecto. El reto fundamental es el de los nuevos modos de organización, diseñando infraestructuras comunes que permitan mayor alcance y extensión.

Con todo, mencionar el término "masas" en educación al margen del sentido cuantitativo que lo acompaña se nos antoja un nexo perogrullesco que, no obstante, arrastra consigo conspicuas exigencias sociales a las que no siempre es posible responder con los medios necesarios. Es claro también que la investigación pedagógica en la órbita de la planificación de sistemas adquiere relevancia una vez captado su propósito, el de estudiar y prever la mejor organización de recursos y medios para la educación de la población en su conjunto; pero sin perder de vista que la solución, antes que escolar y pedagógica, es radicalmente política.

Ahora bien, es muy apropiado reafirmar, en beneficio de la profesión educativa, el reto pedagógico que pone sobre el tapete la educación de masas en la universidad. Por la sencilla razón de que será difícil poder hablar de educación, de formación -o aun de instrucción- sin nuevas estrategias de enseñanza-aprendizaje y de organización escolar e institucional en los centros universitarios, llámese una Facultad, una Escuela Universitaria o, por extensión, una Universidad sin más.

Se sigue de esto, pensamos, que una democracia que avanza, sensible a los requerimientos de la sociedad civil, no puede asistir inerme por más tiempo al pregón de las instancias interesadas en acentuar, míticamente, el tópico de la baja calidad del producto educativo en la sociedad de masas, dejando de apelar, como salida, a la conveniencia de nuevas formas de organización, junto con la revisión fundamental de los procesos formativos en una línea, pedagógicamente ya desarrollada (Martínez Mut, 1997), de reingeniería de procesos para la mejora de la calidad y los servicios.

Nos estamos situando ante el concepto de calidad total en los sistemas universitarios, con irreductibles planes de reflexión e intervención sobre el «estado del arte" en docencia, investigación, gestión y extensión de servicios de variada índole socio-cultural. El desarrollo de sistemas universitarios se complica innecesariamente al margen de espacios coordinados (Touriñán, 1993) que son los que pueden mejorar la oferta de estudios, al tiempo que contribuyen a crear una buena infraestructura de comunicación científica y propician un sistema de gestión integrado. Los servicios de apoyo a la docencia en red para los sistemas universitarios y las redes de ciencia y tecnología por la comunidad científica son alternativas pertinentes de política universitaria. 
5. De la estrategia política a la política estratégica. Definición y desarrollo de PERFILES

Es común y recurrente oír hablar de crisis en la universidad referida, entre otras cosas, al número de alumnos, financiación, planes de estudios, etc. Pero, en el fondo de todas esas crisis puntuales subyace una crisis más global: es la crisis de gestión en la que los responsables de la administración, a cualquier nivel, tienen que definir posiciones, pues, si hay un ámbito de responsabilidad compartido, también hay, como acabamos de apuntar, un conjunto de exigencias diferenciadas.

La perspectiva histórica permite afirmar que la crisis persistente en que vive la universidad desde hace décadas no es una crisis de la universidad como institución, sino la crisis o la decadencia de un determinado modo de hacer universidad. La creación de nuevos usos como problema y solución a la vez. Además, hay que reconocer que en lugar de universidad tendremos "multiversidad", y no ya por la variedad de actividades académicas, sino por el modo de integrarse en el entorno social circundante y la heterogeneidad de su clientela (Sánchez Salorio, 1993; Touriñán, 1997).

Siendo cierto que el espacio y los recursos nos obligan a hablar de universidades orientadas a polos de excelencia, y que en los nuevos modelos universitarios, considerando la masa crítica investigadora, serán necesarios macrocentros integrados que alberguen diversas titulaciones (principio de complementariedad interdisciplinar), no podemos olvidar que todo eso es la consecuencia última de una búsqueda constante para definir y construir estructuras organizativas y de gestión que garanticen de forma eficaz la realización de los valores sustantivos (docencia, investigación, cultura, estudio, profesionalización, desarrollo social productivo) de la universidad. En este sentido, la aplicación del principio de responsabilidad social compartida en los costes, potenciará una planificación y toma de decisiones bien meditadas, con mayores niveles de responsabilidad en la estructura, el proceso y el producto de la enseñanza superior. Nos movemos, por tanto, en unas circunstancias que provocan cambios de mentalidad sobre el presupuesto que alcanza la educación superior.

Quizá, llegó el momento de plantearse sinceramente si la autonomía universitaria reconocida en la Constitución debe permanecer en los términos establecidos en la LRU, preguntándonos si la facilitación de modelos asamblearios favorecen la gestión y una eficaz toma de decisiones. Lo cual no representa ningún cuestionamiento del principio de democratización, ni del de transparencia y participación, sino comprender la necesidad de cauces razonables para que los esfuerzos sinérgicos hagan compatibles la subsidiariedad, la responsabilidad compartida y el rendimiento social de la institución universitaria. Lo que se quiere decir es que no es bueno utilizar el marco legal, preservando el cuerpo de la universidad de modo tal que sea imposible encontrar fórmulas para que la institución rinda cuentas a la sociedad de las funciones que tiene encomendadas y del modo de llevarlas a cabo.

No compartimos una cultura de gobierno de la universidad que haga sinónimo autonomía universitaria e impunidad ya que las responsabilidades en los órga- 
nos de dirección pueden difuminarse hasta extremos irracionales en el imaginario colectivo universitario sin que se garanticen, ni la continuidad temporal de las metas planificadas, ni su consecución. Desde este postulado, la política universitaria fue expansionista sin más freno que el económico y dado que en las universidades, la oferta crea demanda, se tendió a la autorización de nuevas carreras y a la creación de centros sin racionalidad sistémica, socio-económica y académico-organizativa (Rodríguez, Touriñán y Santos, 1996).

Pero además, la Unión Europea se planteó, a partir del Tratado de Maastricht, que uno de los retos más importantes, que tendría que resolver la educación superior y universitaria, sería el que tiene que ver con el desarrollo de sistemas universitarios regionales, lo que provocará en el futuro que las políticas universitarias de las diferentes administraciones tengan que definir los perfiles universitarios para poder desarrollar, con la mejor calidad posible, los sistemas regionales antes comentados.

Estamos en condiciones de afirmar que la fuerza de este planteamiento estriba en volver a hacer aflorar la necesidad de abrir la universidad a la sociedad, procurando nuevas fuentes de financiación y fomentando la captación de recursos externos. Con toda la atención puesta en la docencia, la investigación, la cultura y la profesionalización, que son los objetivos desde los que la universidad puede $\mathrm{y}$ tiene que conectarse con nuevas fuentes financieras para optimizar su propio desarrollo.

Los análisis estructurales de los sistemas educativos permiten afirmar que los títulos académicos son distintos de los puestos profesionales; los títulos académicos habilitan para el ejercicio profesional, pero no garantizan un puesto de trabajo; ni la definición del puesto de trabajo se hace en función de la definición del título, ni el título se define en función de un puesto de trabajo determinado.

La identificación de la titulación como habilitación para el ejercicio profesional no es garantía de puesto de trabajo o ajuste al perfil ocupacional. Es necesario decir frente a esta identificación que, entre formación y empleo, hay un salto estructural que no es consecuencia de un déficit en la formación ni el objetivo que justifica reivindicaciones de nuevas demandas de cualificación en los planes de estudios. Este salto estructural nace de la distancia y diferencia que hay que mantener entre objetivos de la empresa y objetivos del sistema educativo.

Hoy existe amplio acuerdo a la hora de situar, con realismo y responsabilidad, un enfoque político acerca de las posibilidades de la universidad en una sociedad abierta y con un mundo en total interdependencia. Es por ello que la movilidad de las universidades, así como la globalización progresiva del sector en un marco de creciente internacionalización, obliga a pensar en el posicionamiento futuro de las universidades y en las diferentes alternativas estratégicas que se pueden acometer para alcanzar mayores niveles de prestigio y competitividad.

No es otro el escenario en el que cobran sentido interrogantes importantes para proyectar el trabajo necesario desde la esfera comunitaria y la propiamente universitaria. Las preguntas se concretan en torno a múltiples cuestiones, entre otras, al modelo más idóneo para definir el futuro de una institución universitaria específica, los recursos que se precisan para llegar al modelo, las ventajas com- 
petitivas de una universidad frente a otras, la rentabilización de los recursos, la cartera de títulos o servicios universitarios más ajustada a los objetivos de la universidad y su entorno, etc. De igual manera, sería oportuno preguntarse por el significado de una universidad volcada hacia la docencia, o hacia la investigación, con la correspondiente y permanente búsqueda de calidad e identificación de impactos dentro y fuera del sistema. Sin olvidar, por supuesto, un nuevo diseño de las estructuras de gestión y el estudio de las posibilidades que deja abiertas la ley para flexibilizar la actual estructura de gobierno y funcionamiento.

Tal relación de preguntas pueden ser englobadas a través de un interrogante genérico ligado a la identificación del perfil de una universidad. Para Touriñán (1997), una de las posibles vías de solución a estos problemas consiste en establecer mapas de modelos estratégicos a fin de diferenciar grupos homogéneos entre los perfiles universitarios (Coopers \& Lybrand, 1994). El objetivo del mapa de modelos estratégicos es la determinación de una serie de modelos o perfiles que definan comportamientos actuales y previsibles de las universidades. Además, en las universidades, estas alternativas no fueron diseñadas e implantadas por ellas mismas, sino provocadas, entre otras razones, por la creciente presión de la responsabilidad social compartida en los sistemas universitarios, y por la necesidad de la Administración de gestionar la calidad con los recursos públicos.

Dos son las dimensiones a las que atiende básicamente un mapa de modelos estratégicos: a) nivel de diversificación, y b) imagen social de la universidad.

Se entiende por la primera la orientación de una universidad hacia un mayor o menor segmento del mercado. Es conocido que la mayor parte de las universidades se diversificaron en todas las áreas académicas posibles (Ciencias Sociales y Jurídicas, Ciencias de la Salud, Humanidades, Ciencias de la Naturaleza, Tecnología, etc.). El supuesto de partida es que el nivel de diversificación académica se puede desagregar en relación con las titulaciones. De ahí la implantación en algunas universidades de un mayor número de titulaciones en una de esas grandes áreas académicas que en otra.

El concepto de diversificación mide la oferta académica de una universidad y se fundamenta en la idea de segmentación de mercado. Al respecto cabe señalar dos tendencias. Una de ellas hacia la concentración en algunas áreas temáticas y, dentro de éstas, en algunas titulaciones. La orientación a los modelos especializados es aportada por la tendencia a concentrar, en cuyo caso el número de alumnos suele ser menor que en una universidad con mayor diversificación. La otra tendencia tiene su epicentro en la territorialización, atendiendo a la totalidad de la oferta y a la complementariedad de los campus, restringiendo el número de titulaciones por área en cada campus. El objetivo es tener una oferta lo más completa posible para atraer a los universitarios de ese ámbito territorial. Y su orientación es hacia los modelos genéricos.

La segunda de las dimensiones, decíamos, pivota sobre la imagen social de la universidad. Nos referimos con ella a la idea de excelencia que tiene la sociedad de cada institución universitaria atendiendo a los rasgos más característicos de la exclusividad, identidad o perfil, que pueden expresarse, fundamentalmente, por el grado de atracción de sus titulaciones, el grado de dedicación de su profesora- 
do, el nivel de prestigio científico de la universidad, o la atención que presta a sus alumnos en las diversas facetas de su formación.

Es cierto que los modelos estratégicos ayudan a definir el perfil de la universidad y son un elemento a considerar en la financiación y en la racionalización política del sistema. Desde la alternativa genérico-exclusividad y teniendo en cuenta las variables enumeradas, estos modelos permiten la identificación de perfiles de universidad orientados según el caso hacia:

- Captación o no de alumnado de otras universidades.

- Incremento o no de títulos de demanda universitaria.

- Generalización o especialización del perfil.

- Orientación primordial a la docencia.

- Orientación primordial a la investigación.

Se trata de líneas de tendencia que responden a hechos socio-económicos generales, por lo que no extraña su mayor acentuación en la educación universitaria en la etapa post-Maastricht, puesto que en la Unión Europea el reconocimiento de los diplomas de formación constituye el medio que permite conciliar de modo permanente la originalidad de los sistemas nacionales de enseñanza de cada entidad nacional con la movilidad de los ciudadanos que desean dar una dimensión europea a su formación o a sus actividades profesionales. Puede decirse, entonces, que el mutuo desconocimiento entre los sistemas y la ausencia de relaciones de cooperación trabajan en contra de las vías de reconocimiento; por el contrario, la existencia de perfiles de identificación, aproxima a las universidades entre sí, permitiéndoles optimizar sus potenciales de servicio a la comunidad.

Los frutos derivados de este enfoque están ya produciéndose. En esa línea y para fomentar sinergias que faciliten los reconocimientos de diplomas asistimos a la potenciación de cuatro ejes de acción: las redes de información, las redes académicas y profesionales, la adaptación consensuada de las formaciones y la evaluación de la calidad de la formación como instrumentos altamente eficaces para avanzar en los objetivos comunes de la educación universitaria europea.

Completando esta visión, permítasenos la referencia breve y directa a una serie de rasgos que favorecen la identificación de modelos (Touriñán, 1997). Ciñéndonos, en primer lugar, a la identificación de modelos estratégicos genéricos, tenemos en las siguientes algunas de sus notas importantes:

- Están orientados primordialmente a la docencia, intentando potenciar la calidad de la misma, derivando la mayor parte de sus recursos (humanos, tecnológicos, financieros y materiales) hacia la adecuada transmisión de los conocimientos.

- Fortalecen sus colaboraciones con profesores asociados, para disminuir costes fijos de personal docente.

- Desarrollan líneas de investigación especialmente comerciales, para captar recursos financieros que se reinvierten en otras actividades académicas.

- Diseñan estructuras organizativas, asumiendo funciones de gestión en los niveles de vicerrectorado, implantando programas de formación para la gestión. 
JOSÉ M. TOURIÑÁÁN, MIGUEL Á. SANTOS, ANTONIO RODRÍGUEZ Y M. a DEL MAR LORENZO LA FUNCIÓN DE LA UNIVERSIDAD. DE LA ESTRATEGIA POLÍTICA A LA POLÍTICA...

- Potencian los sistemas de información relacionados con la gestión de alumnos y el seguimiento académico de los mismos.

- Fortalecen las actividades de extensión cultural que favorecen la imagen de la universidad en la Comunidad.

- Orientan sus titulaciones y exigencias académicas hacia los sectores medios del mercado, centrándose en las titulaciones consolidadas.

- Fortalecen el primer y segundo ciclo, y en especial las diplomaturas, intentando evitar el desarrollo de cursos master altamente especializados.

- Planifican periódicamente sus necesidades de instalaciones físicas, así como las potenciales inversiones en nuevos activos fijos.

- Se orientan hacia la captación de fondos públicos para equilibrar su incremento progresivo de gastos.

Por su parte, la identificación de modelos estratégicos de concentración y exclusividad pasaría, entre otras, por las notas que a continuación se indican:

- Se orientan hacia la captación de personal académico de muy alta calidad.

- Derivan grandes recursos de la universidad, especialmente tecnológicos, hacia el desarrollo de la investigación, aprovechando ésta para la implantación de programas de cooperación con empresas privadas.

- Interrelacionan la investigación con la docencia, mediante el procesamiento de los resultados de la investigación científica.

- Crean una cultura corporativa de diferenciación.

- Se orientan hacia los criterios de rentabilidad y autofinanciación, así como a establecer mecanismos de autocrítica.

- Se orientan hacia el valor añadido de todos los servicios de la universidad, estableciendo mecanismos que aseguren la calidad de los mismos.

- Desarrollan estrategias de control del crecimiento, para alcanzar una efectiva ratio profesor/alumnos.

- Alcanzan una alta selección académica de sus alumnos, ya sea tendiendo a altas notas medias de acceso a la universidad, o por medio de una mayor exigencia en la actividad académica de los estudiantes.

- Profesionalizan la estructura organizativa de la universidad, dotándola de los adecuados niveles de gestión.

- Proyectan una imagen de calidad y exigencia en el mercado.

- Controlan la diversificación, orientando la oferta académica hacia las titulaciones estrella y los terceros ciclos de calidad.

\section{ACERCA DE LA UNIVERSIDAD QUE VIENE: UNIR VIEJAS FUNCIONES CON NUEVOS RETOS}

El problema de la universidad del futuro no es su democratización o la inserción de nuestra universidad en su medio social, "sino mantener una tensión permanente por la mejora de la calidad" (Quintanilla, 1998, p. 343). Si se nos permite, ¿qué otra utopía pertinente puede movilizar nuestra ilusión y esfuerzo hacia el 
futuro? También nosotros estamos convencidos de que la universidad del siglo XXI se caracterizará por una exigencia de calidad más que de cantidad.

Las funciones de la universidad seguirán coincidiendo, en lo fundamental, con las que afirmaba el maestro Ortega hace más de medio siglo. Funciones, por cierto, concisamente enunciadas en el texto de nuestra Ley de Reforma Universitaria, de 1983, cuya aplicación y desarrollo ha producido, por motivos diversos, no pocas disfunciones y desequilibrios (Ortega Castro, 1996). Pero es la sociedad en la que se inserta la universidad la que irá marcando, sin duda, la (re)definición de funciones en cada situación, aunque sigan pivotando en torno a la creación, desarro1lo, transmisión y crítica de la ciencia, de la técnica y de la preparación para el ejercicio de una profesión, de la creación artística y cultural en general, etc. (Villapalos, 1998).

Sabemos que todas las universidades desarrollan, en mayor o menor proporción, esas funciones genéricas. La historia, condiciones y circunstancias de cada institución modularían, desde luego, el análisis evaluativo acerca de su funcionalidad programática en el servicio a sus "clientes" y a la Comunidad de la que recibe sus recursos.

La definitiva incorporación al proyecto europeo, del que las universidades forman parte, deberá obligar a planes rigurosos, incrustados en un dimensionamiento estratégico de la actividad universitaria a realizar en los próximos años, cuyo epicentro tiene que ser la mejora de la calidad de la enseñanza y de la investigación, al lado de una permanente búsqueda de la eficacia en la prestación del servicio público de la enseñanza superior. Un recurso educativo de la universidad en nuestro entorno se conecta positivamente con la diversidad de la cultura europea.

Además, lo que parece una inevitable tendencia a la globalización social y económica determinará una fuerte apuesta de las universidades por políticas favorables a la internacionalización, de donde se derivará alguna que otra medida del gradiente de competitividad y calidad que es capaz de desarrollar cada institución. La internacionalización universitaria, con sus manifestaciones de trabajo en red y creciente movilidad docente y discente está siendo catapultada gracias a la imparable fuerza de la comunicación electrónica por todo el mundo (cfr. Castells, 1997). Es evidente que la comunicación vía ordenador acompaña ya parte sustantiva del intercambio universitario a nivel internacional.

Tal realidad optimizará en el futuro, aún más, el rol de las universidades como agentes de difusión de la innovación social, puesto que los egresados de sus aulas y laboratorios llevarán a todo tipo de organismos y empresas el mensaje mediático de la cultura de la comunicación. Pero las comunidades virtuales o del ciberespacio no podrán sustituir a la comunidad de profesores y estudiantes de cada universidad, sencillamente porque, como acaba de señalar Rhodes (1998, p. 13) "We need the stimulus of face-to-face communication and shoulder-to-shoulder cooperation in our own campuses". Todo ello ha contribuido a poner de manifiesto el valor del desarrollo y diseño de infraestructuras compartidas y servicios comunes en la solución futura que haga compatible calidad y cantidad. Pues, no 
nos engañemos, la universidad en España es de modelo extenso (esto es, de masas y no masificada).

Entretanto, es preciso que la universidad recupere su protagonismo como agencia capaz de liderar, desde posiciones de independencia, estilos y pautas de pensamiento cultural y científico, susceptibles de influir, críticamente, en la construcción de la nueva sociedad. Su papel como institución que marca la diferencia en una senda de progreso individual y colectivo desde la sociedad civil debe ser apoyado sin reservas por quienes tienen la oportunidad de influir en la orientación de las políticas públicas. Como dijo en Compostela un buen conocedor de la universidad europea, la universidad no existe por ella misma, ni siquiera existe sólo por la ciencia: "L'université existe exclusivement pour la société et particulièrement, mais sans exclusive, compte tenu de sa responsabilité internationale, pour telle société, c'est-à-dire pour l'homme, non pas pour l'homme abstrait, mais bien en premier lieu pour l'homme de cette partie du monde dans laquelle elle se trouve" (Thorens, 1996, p. 44).

Resulta necesario dar credibilidad a una proyección estratégica de la docencia y de su calidad, como exponente principal de compromiso con su principal cliente, el alumno, que es el auténtico protagonista de su propio proceso de aprendizaje, pero al que el profesorado debe atender mejor, sin obsesiones estériles mas con preocupación por utilizar metodologías apropiadas, a fin de no incidir en una información repetitiva y sí en el desarrollo de capacidades, hábitos y actitudes.

La universidad debe reflexionar más acerca de lo que se reconoce como excesiva especialización en los estudios, cuya única meta está siendo la de lograr mayor cercanía al mercado de trabajo, meta algo ingenua porque olvida algo tan sencillo como el hecho de que el empleo no depende, sin más, de la especialización en la formación que reciben los estudiantes. El agobio que producen ciertas situaciones coyunturales no puede nublar la vista sobre el alcance de la misión universitaria en una sociedad digitalizada, donde la educación para el aprendizaje a lo largo de la vida será piedra de toque fundamental. Y los pedagogos tenemos que situarnos en la vanguardia de un proceso que, además, se está enfatizando desde todo tipo de instancias sociales y profesionales.

Esa demanda tiene que ver también con otro importante reto, como son los estudios de tercer ciclo y, en general, todo lo relacionado con las enseñanzas de postgrado, vinculándolas a planes de formación continua, favoreciendo la adaptación al cambio veloz que impone el desarrollo científico y tecnológico, o el que deriva de la irrupción de nuevas profesiones y ocupaciones en un mercado cada vez más abierto, interactivo y, por supuesto, competitivo. Todo lo cual tiene que ver con una función de la universidad, difícil, peculiar y compleja, pero de inequívoca importancia: gestionar el cambio y proyección de sí misma, para seguir fiel a su esencia y cometido histórico. Aunque, ahora, el cometido no sea otro que, recogiendo la brillante idea de Kerr (1995) consolidar la idea de la universidad como "multiversidad".

No menos importante será prestar atención a la creciente demanda de formación cultural en distintos ámbitos y campos de conocimiento, junto con una formación para el ocio y el mayor tiempo libre en un segmento clave y crecien- 
te de la población como es la llamada "tercera edad". Tal función formativa, de extensión y animación cultural, ya ha empezado a ser ejercida, con inusitada expectación, por bastantes universidades dentro de nuestro marco de referencia, rotulando esas actividades de manera diversa ("cuarto ciclo", "universidad de la experiencia", etc.).

Asimismo, tenemos en la formación para la investigación, que debe diferenciarse de la formación para la especialización, otro reto notorio y, por supuesto, funciones a replantear. Existe plena coincidencia en la necesidad de cambiar el enfoque y los estudios del tercer ciclo, devaluados de un tiempo a esta parte en nuestro entorno, con muchos estudiantes matriculados en los programas de doctorado sin vocación auténtica ni preparación adecuada para la realización de tesis doctorales que puedan contribuir a abrir líneas novedosas de indagación científica.

No estaría mal fortalecer programas de doctorado de calidad, empezando por áreas bien dotadas y aquellas otras que se consideren de interés estratégico para la universidad y la sociedad en su conjunto. Sin olvidar que también será una función de la universidad, recuperando parte de un edificante legado, la búsqueda de equilibrios entre la investigación básica y la investigación aplicada, hoy bastante sesgada a favor de ésta, ya que es ahí donde el profesorado ve recompensadas, en general, sus peticiones de financiación para proyectos e iniciativas investigadoras.

Pero si la calidad, finalmente, se asume de verdad como el gran pivote a circundar la siempre delicada relación entre política y universidad, ha de promoverse su articulación procesual de modo tal que, lejos de marginar la mejora real de la docencia, suponga su definitiva expresión de excelencia en la universidad.

Esto exige sistemas de control de la calidad que sean efectivos y, por tanto, creíbles en el seno de la comunidad universitaria, pero no es menos cierto que debe plantearse, al mismo tiempo, en un marco más amplio, estratégicamente proyectado, de cambio en las cuestiones que afectan a los procesos que rigen actualmente la formación, selección y promoción del profesorado.

Sabemos de las razones que han llevado a desequilibrar en favor de la investigación la imprescindible sintonía entre este vector de trabajo universitario y el de la docencia, y por eso no es extraño que hoy surja con fuerza propia la necesidad de replantearse, inserta en el mismo anhelo proclive al objetivo de la calidad, una reflexión profunda sobre la estructura de gobierno universitario. La inquietud social que a veces provoca la constatación de algunos acontecimientos en el seno de la universidad se alienta precisamente con la desidia en el afrontamiento de tamañas cuestiones.

Estamos convencidos, a la luz de todo lo anterior, que la política estratégica de la universidad para los próximos lustros debe empezar por una clara definición de bases, que permita restituir a la razón docente todo su valor en la prestación del servicio universitario a la ciudadanía y ello pone nuevos marcos a la cuestión política de la financiación de la universidad que es una responsabilidad social compartida inherente al derecho a la autonomía universitaria. 
JOSÉ M. TOURIÑÁN, MIGUEL Á. SANTOS, ANTONIO RODRÍGUEZ Y M. ㅁ DEL MAR LORENZO LA FUNCIÓN DE LA UNIVERSIDAD. DE LA ESTRATEGIA POLÍTICA A LA POLÍTICA...

\section{BiBLIOGRAFíA}

Barber, B. R. (1998): A passion for democracy. American essays. Princeton University Press. Princeton, New Jersey,

Beltrán LlaVADOR, F. (1996): “La calidad, más allá de criterios y estándares”, en QuinTás, G. (ed.): Reforma y evaluación de la universidad. Universidad de Valencia. Valencia, pp. 133-164.

Bloom, A. (1987): The closing of the american mind. Simon \& Schuster. New York.

Bobbio, N. y MatTeucci, N. (1982): Diccionario de Política. Siglo XXI Editores. Madrid.

CAPARRós, A. (1997): “Ubicación política universitaria». Diario La Vanguardia, 10 Octubre.

CAstells, M. (1997): La era de la información (vol. 1). La sociedad red. Alianza Editorial. Madrid.

CAVE, M. ET AL. (1997): The use of performance indicators in bigher education. Jessica Kingsley Publications. London.

CNE (1995): Le devenir des diplomes des universités. Comité National d'Évaluation des établissements publics á caractère scientifique, culturell et professionnel. Paris.

COMISIÓN DE LAS COMUNIDAdes EuROPEAs (1990): Colloque sur l'enseignement superieur et 1991: préparons l'an 2000. Université de Sienne.

- (1991): Memorandum sobre la enseñanza superior en la Comunidad Europea. Informe final. COM (91) 349.

- (1992): Report of the bigh performance. Computing and Networking Advisory Committee (Comisión de las Comunidades Europeas). D. G. XIII. Bruselas.

- (1993): Crecimiento, competitividad y empleo. Retos y pistas para entrar en el siglo XXI. Libro Blanco. Fundación Galicia-Europa. Santiago de Compostela.

- (1993a): Orientaciones para la acción comunitaria en el ámbito de la educación y la formación: Informe final. Com. (93), 183 final. Bruselas.

- (1994): La política social europea. Un paso adelante para la Unión. Fundación GaliciaEuropa. Santiago de Compostela.

CONSEJO DE UNIVERSIDADES (1995): Estadistica de la matrícula universitaria. Curso 1994-95. Secretaría del Consejo de Universidades. Madrid.

- (1996): Plan Nacional de Evaluación de la Calidad del Sistema Universitario: Guía de Evaluación. Consejo de Universidades. Madrid.

- (1997): Informe sobre los resultados de la primera convocatoria del Plan Nacional de Evaluación de la Calidad de las Universidades. Secretaría General del Consejo de Universidades. Madrid.

COOPERS \& LYBRAND (1994): Modelos estratégicos (documento policopiado, diciembre). Madrid.

Cortina, A. (1997): Ciudadanos del mundo. Hacia una teoría de la ciudadanía. Alianza Editorial. Madrid.

E.O.L.A.S. (1992): Memorandum on bigher education in the european community. European Association of Deans of Science. Salamanca.

GARCIA, P. ET AL. (1995): “Experimenting institutional evaluation in Spain». Higher Education Management, 7, 1, pp. 101-118.

HunTINGTON, S. (1997): El choque de civilizaciones y la reconfiguración del orden mundial. Paidós. Barcelona.

HuSEN, T. (1994): The role of the University: a global perspective. The United Nations University. Tokio.

JEREZ MIR, R. (1997): «La universidad en la encrucijada: universidad dual o universidad democrática y de masas". Revista de Educación, 314, pp. 137-156.

KERR, C. (1995): The uses of the university. Harvard University Press (edición actualizada; primera edición en 1963). Cambridge. Massachusetts. 
JOSÉ M. TOURIÑÁN, MIGUEL Á. SANTOS, ANTONIO RODRÍGUEZ Y M. DEL MAR LORENZO LA FUNCIÓN DE LA UNIVERSIDAD. DE LA ESTRATEGIA POLÍTICA A LA POLÍTICA...

Malosse, H. (1994): Europa a su alcance. Fundación Galicia-Europa. Santiago de Compostela. Martínez Mut, B. (1997): Calidad y Educación. Un enfoque de ingeniería. Tirant lo Blanch. Valencia.

Matteucci, N. (ed.) (1991): L'Università nel mondo contemporaneo. Bompiani. Milán.

Michavila, F. (1998): "Evaluación institucional para la mejora de la actividad académica". Ponencia al Seminario Internacional sobre dirección estratégica y calidad de las universidades (19-21 de enero). Universidad Politécnica de Cataluña (policopiado). Barcelona.

Neave, G. (1995): “Las políticas de calidad: desarrollos en enseñanza superior en Europa occidental. Revista de Educación, 308, pp. 7-29.

O'BRIEN, G. E. (1998): All the essentials half-truths about bigher education. The University of Chicago Press. Chicago.

ORDEN, A. DE LA (1985): "Modelos de evaluación universitaria». Revista Española de Pedagogia, 169-170, pp. 521-537.

- (1993): “Calidad y evaluación de la enseñanza universitaria”. En Varios: La Universidad ante el $V$ Centenario, Actas del Congreso Internacional de Universidades. Editorial Complutense. Madrid, pp. 531-539.

Ortega Castro, V. (1996): “Funciones y disfunciones de las universidades españolas". Ponencia al Congreso $A$ Universidade e o seu Futuro. V Centenario de la Universidad de Santiago de Compostela (12-14 de abril) (policopiado).

Ortega y Gasset, J. (1975): Misión de la Universidad y otros ensayos. Publicaciones de la Revista de Occidente. Madrid.

Pérez Díaz, V. (1997): La esfera pública y la sociedad civil, capítulo 4. Taurus. Madrid.

Quintanilla, M. A. (1998): "Nuevas ideas para la universidad". En Luxán, J. M. (ed.): Política $y$ reforma universitaria. Cedecs. Barcelona, pp. 341-351.

Rhodes, F. H. T. (1998): "The university and its critics". En Bowen, W. G. \& SHapiro, H. T.: Universities and their Leadership. Princeton University Press. Princeton. New Jersey, pp. 3-14.

Rodríguez, A., Touriñán, J. M. y SANTos, M. A. (1996): "La lógica de las decisiones en la construcción de sistemas educativos: estudio de un caso". Revista Española de Pedagogía, 54, 205, pp. 511-534.

RODRÍGUEZ ESPINAR, S. (1997): “La evaluación institucional universitaria”. Revista de Investigación Educativa, 15, 2, pp. 179-214.

Rodríguez NAVARRo, A. (1994): "La reforma de la universidad". Boletín de la Institución Libre de Enseñanza, 20.

SÁNChez SAlorio, M. (1993): Universidade. Multiversidade. Megaversidade. Universidad de Santiago de Compostela. Santiago de Compostela.

- (1996): "La universidad ante el cambio de paradigma. Nuevos retos, nuevas respuestas". Ponencia al Congreso A Universidade e o seu Futuro. V Centenario de la Universidad de Santiago de Compostela (12-14 abril) (policopiado).

Santos Rego, M. A. et al. (1996): "Educación y cultura de masas". En Escámez, J. (ed.): Acción educativa y comunicación social. Tirant lo Blanch. Valencia, pp. 113-158.

Santos Rego, M. A. (ed.) (1997): Política educativa en la Unión Europea después de Maastricht/Educational policy in the European Union after Maastricht (ed. bilingüe español-inglés). Escuela Gallega de Administración Pública. Santiago de Compostela.

- (1997a): «Educación y Principio de Subsidiariedad: un nuevo marco relacional EstadoComunidades Autónomas en España". En SAnTos Rego, M. A. (ed.): Política Educativa 
JOSÉ M. TOURIÑÁN, MIGUEL Á. SANTOS, ANTONIO RODRÍGUEZ Y M. ${ }^{2}$ DEL MAR LORENZO LA FUNCIÓN DE LA UNIVERSIDAD. DE LA ESTRATEGIA POLÍTICA A LA POLÍTICA...

en la Unión Europea después de Maastricht. Escuela Gallega de Administración Pública. Santiago de Compostela, pp. 171-189.

TAYLoR, Ch. (1993): El multiculturalismo y la politica del reconocimiento. Fondo de Cultura Económica (FCE). México.

Thorens, J. (1996): “Role et mission de l'université à l'aube du XXI ${ }^{\text {ème }}$ siècle». Ponencia al Congreso $A$ Universidade e o seu Futuro. V Centenario de la Universidad de Santiago de Compostela (12-14 abril) (policopiado).

Tiana Ferrer, A. (1998): "La demanda de estudios universitarios y el acceso a la universidad". En LuXÁn, J. M. (ed.): Política y reforma universitaria. Cedecs. Barcelona, pp. 177-189.

TOURIÑÁn, J. M. (1993) (dir.): Avances no desenvolvemento do sistema universitario de Galicia. Xunta de Galicia. Santiago de Compostela.

- (1993a): “Un reto de los sistemas universitarios: los espacios de coordinación”. En Varios: La Universidad ante el V Centenario, Actas del Congreso Internacional de Universidades. Editorial Complutense. Madrid, pp. 497-507.

- (1995): «La descentralización educativa. Análisis desde la experiencia universitaria en Galicia". Foro Internacional de Escuela, Familia y Sociedad. Instituto de Fomento e Innovación Educativa. México, D.F.

- (1996) (dir.): Sistema universitario de Galicia. Fundamentos e desenvolvemento legal. Xunta de Galicia. Santiago de Compostela.

- (1996a): "Nuevos desafíos en el desarrollo universitario: el efecto Maastricht". En S.E.P./U.P.V.: Innovación Pedagógica y Políticas Educativas. XI Congreso Nacional de Pedagogía. San Sebastián/Donostia, tomo III, pp. 165-186.

- (1997): La consolidación del sistema universitario y de la comunidad científica en Galicia. Propuestas de análisis. Grafinova. Santiago de Compostela.

- (1997a): «La búsqueda de calidad en el desarrollo universitario y la definición de perfiles". Revista Galega de Cooperación Científica Iberoamericana, 4, pp. 40-61.

- (1997b): "La educación universitaria después del tratado de Maastricht". En SANTOS REgo, M. A. (ed.): Política educativa en la Unión Europea después de Maastricht. Escuela Gallega de Administración Pública. Santiago de Compostela, pp. 193-211.

- (1997c): «Directrices comunitarias de la educación universitaria: crisis de la institución y estrategias de innovación. Revista Galego-Portuguesa de Psicoloxía e Educación, 1, 1, pp. 9-32.

UnesCo (1995): Documento de política para el cambio y el desarrollo en la educación superior. Unesco. París.

Universidad Politécnica de Cataluña (1997): Calidad al servicio de la sociedad. U.P.C. Barcelona.

URIEL, E. ET AL. (1997): Las cuentas de la educación en España y sus comunidades autónomas: 1980-1992. Fundación Argentaria y Visor. Madrid.

VÁzQuez Gómez, G. (1993): «El papel del profesor universitario en una sociedad post-industrial. En VARIOS: La Universidad ante el $V$ Centenario, Actas del Congreso Internacional de Universidades. Editorial Complutense. Madrid, pp. 699-704.

VILLANUEVA, D. (1997): “A sociedade civil, entre as dictaduras residuais e a democracia real". XXII Conferencia Mundial da Sociedade Internacional para o desenvolvemento (21-24 mayo). Santiago de Compostela (policopiado).

VILlapalos, G. (1998): "El futuro de la Universidad”. En LuXÁn, J. M. (ed.): Política y reforma universitaria. Cedecs. Barcelona, pp. 333-340.

VORBECK, M. (1998): "Quality assessment in higher education. A european perspective". Ponencia al Seminario Internacional sobre dirección estratégica y calidad de las 
JOSÉ M. TOURIÑÁN, MIGUEL Á. SANTOS, ANTONIO RODRÍGUEZ Y M.르 DEL MAR LORENZO LA FUNCIÓN DE LA UNIVERSIDAD. DE LA ESTRATEGIA POLÍTICA A LA POLÍTICA...

universidades (19-21 enero). Universidad Politécnica de Cataluña (policopiado). Barcelona.

WOOT, P. DE (1998): "Managing strategic change in university". Ponencia al Seminario Internacional sobre dirección estratégica y calidad de las universidades (19-21 enero). Universidad Politécnica de Cataluña (policopiado). Barcelona. 